Check for updates

Cite this: J. Mater. Chem. A, 2021, 9, 8401

\section{Bottom-up assembly of bimetallic nanocluster catalysts from oxide-supported single-atom precursors $\dagger$}

\author{
Bidyut B. Sarma, (D) †' Giovanni Agostini, (D) ${ }^{\mathrm{b}}$ Marcos G. Farpón, ${ }^{\mathrm{c}}$ Carlo Marini, ${ }^{\mathrm{b}}$ \\ Norbert Pfänder ${ }^{d}$ and Gonzalo Prieto iD *ac
}

\begin{abstract}
The precise synthesis and stabilization of oxide-supported bimetallic clusters in the low-to-sub nanometer size regime is highly relevant in various fields, from optics and sensing to electrochemistry and catalysis. In surface-driven phenomena such as catalysis, avoiding metal segregation and agglomeration is essential for performance and stability under relevant operation conditions. Here we show how high-temperature oxidative crystal redispersion and oxide atom-trapping phenomena provide a widely valid route towards atomically dispersed bimetallic precursors which, upon reductive metal agglomeration, result in very small, uniformly sized and remarkably stable bimetallic clusters. For a PdPt/MgO system, oxidative redispersion leads to isolated $\mathrm{Pd}$ and $\mathrm{Pt}$ atoms stabilized by the $\mathrm{MgO}$ support up to overall surface metal contents of ca. $1.0 \mathrm{Mat}_{\mathrm{at}} \mathrm{nm}^{-2}$, beyond which loading, atom-trapping oxide sites become exhausted and metal aggregation sets in. On the contrary, more conventional, milder-temperature activation protocols lead to significant metal segregation and markedly bimodal particle size populations already from comparatively lower metal contents. As proven by in situ X-ray absorption spectroscopy and atomicresolution STEM microscopy, the stabilization of isolated $\mathrm{Pd}$ and $\mathrm{Pt}$ cations within nanometer distances on the common $\mathrm{MgO}$ support is essential for the synthesis of ca. $1 \mathrm{~nm}$ bimetallic PdPt clusters by reductive agglomeration. The uniformly sized PdPt aggregates developed on $\mathrm{MgO}$ from single-atom precursors display a notably higher activity for the oxidative activation of methane with carbon dioxide (dry reforming) at $923 \mathrm{~K}$ compared to analogue materials synthesized via milder calcination/reduction protocols. Moreover, despite their high surface-to-volume ratio, the small bimetallic clusters in the former display an outstanding stability against metal agglomeration under the demanding reaction conditions, likely as a result of a lower driving force for Ostwald ripening growth processes. The synthesis concept, which is amenable to other combinations of $4 d$ and $5 d$ transition metals, contributes to the rationalization of the possibilities and bounds of oxidative metal redispersion phenomena and provides a technically simple and potentially general route to high-temperature stable, (sub)nanometer bimetallic clusters for catalysis and other applications where bimetallic effects and thermal stability are of importance.
\end{abstract}

Received 15th January 2021 Accepted 3rd March 2021

DOI: $10.1039 / \mathrm{d} 1 \mathrm{ta00421b}$ rsc.li/materials-a

\section{Introduction}

Supported bimetallic nanoclusters are important functional materials in areas spanning from photoluminescence, to sensing, electrochemistry and catalysis. ${ }^{1-7}$ As solid catalysts, synergistic effects ascribed to the concomitant presence of two metals within nanosized aggregates, nanoclusters or nanoparticles, have been shown to unlock favourable, in some instances unique, catalytic performances in a broad range of conversion processes. ${ }^{7-10}$ Pioneering work in this area by Sinfelt et al. in alkane dehydrogenation and hydrogenolysis reactions $\mathrm{s}^{11,12}$ was followed by a surge of studies which demonstrated the potential of bimetallic catalysts in a wide array of reactions, from oxidation, ${ }^{13-15}$ to hydrogenation, ${ }^{16-20}$ reforming, ${ }^{21}$ biomass conversion, ${ }^{5} \mathrm{C}-\mathrm{C}$ coupling, ${ }^{22}$ or acetoxylation catalysis. ${ }^{23}$ The 
distinctive catalytic properties of bimetallics compared to their monometallic counterparts has been associated to factors such as differences in lattice strain and surface topology, intermetallic charge transfer phenomena, or atomic-scale surface dilution phenomena of one metal within a matrix of second metal (also referred to as single-atom alloys). ${ }^{\mathbf{9 , 2 4 , 2 5}}$ Most of these effects are more prominent at small particle sizes, e.g. in the low- and sub-nm regime, where a higher fraction of highly reactive, coordinatively unsaturated, surface sites (cus) often dominates catalytic performance.

With the aforementioned encouraging application background, the interest in developing effective, precise and scalable synthesis strategies for supported bimetallic nanoclusters and nanoparticles has witnessed a notable thrust over the last decades. Colloidal methods rooted in the co-reduction of precursor salts in solution, in the presence of organic surface capping agents, is a widely extended synthesis approach. ${ }^{\mathbf{1 0 , 2 6 , 2 7}}$ However, despite their capacity to induce monodispersity, colloidal methods show limitations to produce particles of sizes approaching the sub-nm regime $e . g$. $<2 \mathrm{~nm}$. In order to produce very small bimetallic clusters on solid surfaces, methods based on the adsorption of molecular metal complexes from solution, followed by their reductive decomposition, have also been intensively explored. ${ }^{28}$ These methods face limitations connected to the need for suitable molecular precursors showing adequate adsorption energies on a common carrier as well as comparable thermal stability to prevent step-wise decompositions and thus metal segregation. Scalable synthesis routes which utilize less complex and more widely available organic and inorganic metal precursors are preferred for catalytic applications.

The synthesis of ultrasmall $(\sim 1-2 \mathrm{~nm})$ bimetallic nanoparticles was demonstrated by Regalbuto and coworkers by strong electrostatic adsorption from amine metal precursors by careful adjustment of the electrostatic charges of the support's surface and the metal precursors in solution, respectively. ${ }^{29}$ In a similar approach, Ding et al. adsorbed double complex metal salts, created via electrostatic interaction of oppositely charged inorganic salts, on a silica carrier. ${ }^{30}$ Subsequent thermal decomposition led to bimetallic nanoparticles of $c a .3 \mathrm{~nm}$ in size. Methods based on electrostatic adsorption are thus promising for the synthesis of very small bimetallic aggregates, although the overall metal loading is typically limited by the adsorption capacity of the oxide support. ${ }^{29}$ Other approaches, including radiolysis, ${ }^{31}$ sonochemical synthesis ${ }^{32}$ and step-wise ion-exchange and framework demetallation of zeotype solid precursors $^{33}$ have also been demonstrated for the synthesis of supported bimetallic nanoparticles in the $<2 \mathrm{~nm}$ size range.

Recently, the interest in oxide-supported atomically dispersed metals in catalysis, often referred to as single-atom catalysts, ${ }^{\mathbf{3 4}, 35}$ has spurred research on synthesis routes leading to atomic-level dispersion of metals on oxide carriers. Processes of oxidative crystal disruption and re-dispersion, coupled by atom trapping on oxide surfaces, offer a promising strategy to atomically disperse transition metals - which form highly mobile (sub-)oxides at high temperatures - on suitable carriers. ${ }^{36-38}$ Here we show that two metals can be oxidatively co- dispersed, down to the atomic level, on a common oxide support. The atomic-level structure and reduction behaviour of the resulting bimetallic materials is investigated with ScanningTransmission Electron Microscopy (STEM), and in situ X-ray absorption spectroscopy. Metal reduction with hydrogen leads to very small bimetallic clusters, stabilized on a $\mathrm{MgO}$ carrier, which can only be achieved from atomically dispersed precursors and show remarkable stability under harsh reaction conditions in the dry reforming of methane with $\mathrm{CO}_{2}$. Moreover, the synthesis route is amenable to different combinations of $4 \mathrm{~d}$ and $5 \mathrm{~d}$ transition metals.

\section{Experimental}

\section{Materials}

Metal precursors platinum(II) acetylacetonate (97\%), palladiu$\mathrm{m}$ (II) acetylacetonate (99\%), iridium(III) acetylacetonate (97\%), as well as solvent acetone (>99.9\%) were purchased from SigmaAldrich (Merck) and used as received. For the synthesis of $\mathrm{MgO}$, $\mathrm{Mg}\left(\mathrm{OCH}_{3}\right)_{2}$ (7-8 wt\% solution in methanol) was purchased from Alfa-Aesar and technical-grade toluene was in-house distilled. Bulk PdO (99.97\%) and $\mathrm{PtO}_{2}$ hydrate ( $\left.\geq 99.9 \%\right)$ were purchased from Sigma-Aldrich (Merck).

\section{Synthesis of MgO support material}

A MgO xerogel was synthesized employing magnesium(II) methoxide (7-8 wt\% in methanol) as precursor. ${ }^{39} 100 \mathrm{~mL}$ of the $\mathrm{Mg}\left(\mathrm{OCH}_{3}\right)_{2}$ stock solution was further diluted in $1 \mathrm{~L}$ toluene. Then Milli-Q water was dropwise added to the solution until complete precipitation and the resulting suspension was stirred overnight. The resulting gel was collected by centrifugation (9000 rpm), dried at $298 \mathrm{~K}$ in air overnight and finally at $353 \mathrm{~K}$ for 12 hours. The solid obtained was ground, sieved (200-300 $\mu \mathrm{m})$ and calcined under synthetic air flow $\left(50 \mathrm{~mL} \mathrm{~min}^{-1}\right)$ at 773 $\mathrm{K}$ in a packed-bed reactor for 5 hours (heating ramp from room temperature of $2 \mathrm{~K} \mathrm{~min}^{-1}$ ).

\section{Synthesis of bimetallic atomically dispersed precursors}

Metals were dispersed on the surface of the MgO support via oxidative redispersion. Equimolar amounts of the corresponding metal acetylacetonate precursors required to achieve a preset total metal content $\left(0.2-4.0 \mathrm{M}_{\mathrm{at}} \mathrm{nm}^{-2}\right)$ after air-annealing at $1073 \mathrm{~K}$, were dissolved in $40 \mathrm{~mL}$ of acetone. These metal contents corresponded to overall weight loadings in the range of $0.3-5.4 \mathrm{wt} \%$ (Table S1 in the ESI $\dagger$ ). Then the MgO support material (previously ground to a fine powder) was suspended in the solution and the slurry sonicated. Solvent was then evaporated to dryness in a rotary evaporator under vacuum (in the range of 430-100 mbar) at $313 \mathrm{~K}$ and the solid was dried at $353 \mathrm{~K}$ overnight. The dried impregnate was then transferred to an uncapped porcelain crucible and heated to $1073 \mathrm{~K}$ under a stagnant air atmosphere in a muffle oven for 10 hours, using a temperature ramp of $2 \mathrm{~K} \mathrm{~min}^{-1}$. Materials were denoted as $x \mathrm{M}_{1} \mathrm{M}_{2} / \mathrm{MgO}$, where $x$ indicates the surface-specific metal content corresponding to the state after air-annealing at $1073 \mathrm{~K}$, 
and $\mathrm{M}_{1}$ and $\mathrm{M}_{2}$ represent the two metals incorporated, in an equimolar content, on $\mathrm{MgO}$.

\section{Synthesis of MgO-supported bimetallic nanoclusters}

Bimetallic nanoclusters were generated on the surface of the $\mathrm{MgO}$ carrier by reduction of the corresponding oxide precursors under flow of $20 \% \mathrm{H}_{2} / \mathrm{N}_{2}$ gas mixture. Reduction was performed in a vertically aligned packed-bed reactor using a gas space velocity of $100 \mathrm{~mL} \mathrm{~g}^{-1} \mathrm{~min}^{-1}$. The temperature was increased from RT to $673 \mathrm{~K}$ for Pt-Pd and $773 \mathrm{~K}$ for Pt-Ir at a constant heating rate of $2 \mathrm{~K} \mathrm{~min}^{-1}$. In order to elucidate the significance of the atomic dispersion of the oxidic precursors for the synthesis of the bimetallic nanoclusters, additional materials were also synthesized from precursors which had not been submitted to the high-temperature oxidative crystal redispersion treatment. In one instance, the acetylacetonate metal precursors were directly decomposed on the surface of the MgO support, followed by metal reduction, by treating the dried impregnate under flow of $20 \% \mathrm{H}_{2} / \mathrm{N}_{2}$ gas mixture as described above. Alternatively, the impregnated acetylacetonate precursors were decomposed by calcination in air flow at temperatures of $623 \mathrm{~K}$ or $773 \mathrm{~K}$, followed by reduction of the corresponding oxidic precursors in flow of $20 \% \mathrm{H}_{2} / \mathrm{N}_{2}$ as detailed previously.

\section{$\mathbf{N}_{2}$ physisorption}

Nitrogen physisorption isotherms were recorded at $77 \mathrm{~K}$ using a Micromeritics 3Flex v4.04 instrument. Prior to the measurement, samples were dried at $423 \mathrm{~K}$ under vacuum for $5 \mathrm{~h}$. Specific surface areas were derived using the BET method in the relative pressure $\left(P / P_{0}\right)$ range from $0.05-0.30$.

\section{Powder X-ray diffraction (XRD)}

Powder X-ray diffraction patterns were collected on a Stoe STADI $P$ transmission diffractometer equipped with a primary Ge (111) monochromator and a position-sensitive detector. Data were collected using MoK $\alpha 1$ radiation $(\lambda=0.7093 \AA)$ in the $2 \theta$ range between 5 and $50^{\circ}$ with a step width of $0.015^{\circ}$ and a measuring time per step of $20 \mathrm{~s}$. Samples were filled into glass capillaries ( $\varnothing$ $0.5 \mathrm{~mm})$ and 8 consecutive full scans were collected and summed-up. Acquired patterns were evaluated qualitatively by comparison with entries from the ICCD PDF-2 powder pattern database.

\section{CO chemisorption}

CO chemisorption was used to quantify metal surface atoms after exposure of selected catalysts to dry reforming reaction conditions for $70 \mathrm{~h}$ (vide infra) and thus determine metalsurface specific reaction rates (TOF). Experiments were performed in a Quantachrome Autosorb-1C equipment using the double isotherm method. Prior to chemisorption, catalyst samples $(150 \mathrm{mg}$, pellet size of $200-400 \mu \mathrm{m})$ were treated in situ in $\mathrm{H}_{2}$ flow $\left(25 \mathrm{~mL} \mathrm{~min}{ }^{-1}\right)$ at $673 \mathrm{~K}$ for $5 \mathrm{~h}$ to reverse any surface metal reoxidation due to handling of the catalyst in air after reactor off-loading. Then, samples were degassed at $1.3 \mathrm{~Pa}$ for $2 \mathrm{~h}$ at the reduction temperature, and the temperature was lowered to $308 \mathrm{~K}$. Pure $\mathrm{CO}$ was admitted and the first chemisorption isotherm (total CO uptake) was recorded at $308 \mathrm{~K}$. After evacuation at $308 \mathrm{~K}$, a second isotherm (reversible CO uptake) was registered. The amount of strongly chemisorbed $\mathrm{CO}$ was then obtained by subtracting the two isotherms. The CO pressure range studied was $0.5-11.0 \times 10^{4} \mathrm{~Pa}$. A CO : $\mathrm{M}_{\mathrm{S}}$ surface chemisorption stoichiometry of $1: 1$ was considered.

\section{Scanning-transmission electron microscopy (STEM) and energy-dispersive X-ray (EDS) spectroscopy}

High-angle annular dark-field scanning-transmission microscopy (HAADF-STEM) experiments were performed using a Csprobe-corrected Hitachi HD-2700 dedicated ScanningTransmission Electron Microscope equipped with a cold fieldemission gun and two EDAX Octane T Ultra W EDS detectors and operated at $200 \mathrm{kV}$. Prior to observation, finely ground powder samples were dry-cast on $\mathrm{Cu}$ grids coated with a holey carbon film. Metal nanoparticle size distributions were generated from populations of at least 100 particles coming from different micrographs. The following eqn (1) was used to estimate the average number of atoms from the number-averaged metal cluster size, assuming a spherical cluster shape:

$$
N_{\text {atom }}=\frac{N_{\mathrm{Av}} 4 / 3 \pi(d / 2)^{3}\left(y_{1} / \rho_{1}+y_{2} / \rho_{2}\right)^{-1}}{10^{21} \times\left(\mathrm{AW}_{1} x_{1}+\mathrm{AW}_{2} x_{2}\right)}
$$

where $N_{\mathrm{Av}}$ represents Avogadro's number, $d$ stands for the average nanocluster size in $\mathrm{nm}, x_{i}$ and $y_{i}$ are the molar and mass fractions, respectively, and $\rho_{i}$ and $\mathrm{AW}_{i}$ stand for the density (g $\left.\mathrm{cm}^{-3}\right)$ and atomic weight $\left(\mathrm{g} \mathrm{mol}^{-1}\right)$ of the $i$-metal in the bimetallic clusters.

\section{X-ray absorption spectroscopy (XAS)}

X-Ray absorption spectra were recorded at the Pd K-edge (24.350 $\mathrm{keV}$ ) and $\mathrm{Pt}_{3}$-edge (11.564 keV), respectively, at the CL/ESS beamline station (BL22) of the ALBA synchrotron light source, Barcelona (Spain). ${ }^{40}$ The X-ray beam was monochromatized using a (311) double crystal monochromator. Ex situ measurements on oxidic catalyst precursors were performed at room temperature either in transmission or fluorescence modes, depending on the weight metal loading on the sample. For transmission measurements, powder samples were pressed into pellets $(\varnothing=31 \mathrm{~mm})$ with optimized thickness after dilution, when required, with boron nitride. Absorption spectra were registered with ion chambers filled with appropriate gases to absorb $15 \%$ and $80 \%$ in the $I_{0}$ and $I_{1}$ at the different photon energies, respectively. For fluorescence measurements, sample pellets were mounted in a $45^{\circ}$ angle with respect to the incoming beam and the spectra were registered using a fluorescence solid-state Silicon Drift Detector placed with an angle of $90^{\circ}$ respect to the incoming beam. High-purity bulk metal oxide reference materials were measured in transmission mode as described above.

In situ X-ray Absorption Near Edge Structure (XANES) experiments were performed to follow the reduction behaviour of selected bimetallic oxide precursors. The powder samples were hand-pressed into a pellet sample holder and mounted in 
a multipurpose gas-solid cell, previously described by Guilera et $a l .{ }^{41}$ and equipped with a gas dosing system with Bronkhorst mass flow controllers and temperature control. The sample pellet was oriented in a $45^{\circ}$ angle with respect to the incoming beam and the measurements were performed in fluorescence mode. The cell was purged with $20 \% \mathrm{H}_{2} / \mathrm{He}$ at room temperature and the total gas flow was then set to $100 \mathrm{~mL} \mathrm{~min}^{-1}$. Next, the temperature was increased from $298 \mathrm{~K}$ to $773 \mathrm{~K}$ at a heating rate of $5 \mathrm{~K} \mathrm{~min}^{-1}$. XANES spectra were collected at the corresponding metal edge energy with an energy resolution of $1 \mathrm{eV}$ and a temperature resolution of one full spectrum every $6 \mathrm{~K}$ of temperature change during heating ramps. EXAFS spectra were collected at RT both prior to and after the reduction treatment. For all bimetallic samples, the procedure was repeated at the corresponding X-ray absorption energy edge of each of the two metals.

Data reduction and extraction of the $\chi(k)$ function has been performed using the Athena code (version 0.9.26), ${ }^{42}$ while EXAFS data analysis was performed using the Artemis (Demeter software package). For data fitting a $k$-range $4-10.8 \AA^{-1}$ was chosen with a Rbkg value of 1 . Temperature-resolved in situ XANES spectra have been analysed using the MCR-ALS (Multivariate Curved Regression with Alternative Least Squares) chemometric method $^{\mathbf{4 3 4} 4}$ applying the algorithm developed by Jaumot, Tauler and co-workers. ${ }^{45}$

\section{Catalysis}

Catalysis experiments have been performed in a PIDEng microactivity setup, equipped with a tubular 316 stainless-steel reactor (ID $=7.8 \mathrm{~mm}$ ) placed inside an annular oven. Gas feeds i.e. $\mathrm{Ar}\left(99.99 \%\right.$, as dilutant) and a certified $\mathrm{CH}_{4} / \mathrm{CO}_{2}$ mixture (60/ $40 \mathrm{v} / \mathrm{v} \%$, Linde) were fed using calibrated mass flow controllers. About $200 \mathrm{mg}$ of catalyst $(200-400 \mu \mathrm{m})$ were diluted with $\mathrm{SiC}$ granules $(600-800 \mu \mathrm{m})$ to achieve an isothermal packed bed with an overall volume of $1.9 \mathrm{~cm}^{3}$. The catalyst was reduced prior to reaction at $673 \mathrm{~K}\left(2 \mathrm{~h}\right.$, heating rate of $\left.2 \mathrm{~K} \mathrm{~min}^{-1}\right)$ under

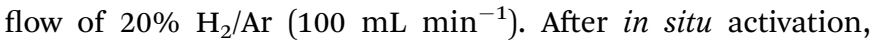
reaction tests were performed at a constant temperature of 923 $\mathrm{K}$ and atmospheric pressure, feeding a reactant gas mixture with a molar composition $\mathrm{Ar} / \mathrm{CH}_{4} / \mathrm{CO}_{2}$ : 4.4/1.5/1.0 at a weight hourly space velocity (WHSV) of $6.9 \mathrm{~h}^{-1}$. The gas reactor outlet was analysed periodically in a SCION 456 gas chromatograph, equipped with a TCD detector and Scionbond-Q and Molsieve $5 \mathrm{~A}$ capillary columns using $\mathrm{H}_{2}$ as a carrier gas. Hydrogen was quantified offline in a Bruker 450GC Rapid Refinery Gas Analyzer (RRGA), equipped with two TCD channels and Molsieve 5A/Hayesep-Q and Molsieve 13X/Hayesep-Q columns and $\mathrm{N}_{2}$ as carrier gas. Gas samples had been collected at the reactor outlet using sealed gas transfer bags after $30 \mathrm{~h}$ on-stream. Forward methane conversion rates have been corrected by an approach-to-equilibrium term $(\eta)$ defined as: ${ }^{46}$

$$
\begin{gathered}
r_{\mathrm{CH}_{4}}^{\mathrm{fw}}=r_{\mathrm{CH}_{4}}^{\mathrm{obs}} /(1-\eta) \\
\eta=\frac{\left[P_{\mathrm{H}_{2}}\right]^{2}\left[P_{\mathrm{CO}}\right]^{2}}{\left[P_{\mathrm{CH}_{4}}\right]\left[P_{\mathrm{CO}_{2}}\right]} \times \frac{1}{K_{\mathrm{eq}}}
\end{gathered}
$$

where $r^{\mathrm{fw}}$ and $r^{\mathrm{obs}}$ represent forward and observed methane conversion rates, respectively, the reaction equilibrium constant $\left(K_{\text {eq }}\right)$ was determined via simulation with an equilibrium Gibbs reactor module in Aspen-Hysys (AspecTech) and the Peng-Robinson equation of state, and $P_{\mathrm{i}}$ stands for the average partial pressure of the $i$-compound within the catalyst bed.

\section{Results and discussion}

\section{Synthesis of supported bimetallic nanoparticles}

Palladium and platinum were dispersed on a high-surface $\mathrm{MgO}$ support material, previously obtained via a sol-gel route. The nanostructure of this material was predominantly composed by rod-like nanocrystals, which exposed a specific surface area of $250 \mathrm{~m}^{2} \mathrm{~g}^{-1}$ (Fig. S1 in the ESI $\dagger$ ). Bimetallic catalysts were synthesized by dispersion of the corresponding acetylacetonate metal precursors on the as-synthesized $\mathrm{MgO}$ carrier, in a $1: 1$ atomic ratio, followed by different thermal activation treatments to decompose the organometallic precursors and reduce metals to their elemental state. Fig. 1 summarizes those thermal activation treatments applied to produce different materials starting from a common dry impregnate.

Powder X-ray diffraction was applied to assess the presence of crystalline metal phases on materials with different surfacespecific metal content and thermal activation history. Fig. 2 shows the diffraction patterns recorded for $1.0 \mathrm{PdPt} / \mathrm{MgO}$ catalysts derived from a single impregnate precursor through different thermal activation routes. The dry impregnate showed diffractions corresponding to the $\mathrm{MgO}$ carrier $(F d 3 m)$, indicating the amorphous character of the acetylacetonate metal precursors deposited on the support surface. Following the decomposition of the supported metal precursors and metal reduction, in a single activation step under a reductive $\left(20 \% \mathrm{H}_{2} /\right.$ $\mathrm{N}_{2}$ ) atmosphere, additional diffractions emerged which could be indexed to a fcc metallic phase $(F m \overline{3} m)$, indicating metal reduction. Close examination of the emerging diffraction lines enabled their assignment to metallic Pt (Fig. S2†). Even though the asymmetric skew of the diffraction lines towards lower $d$ spacings does not enable to rule out contributions from PdPt alloy or Pd crystallites, this result clearly points to metal segregation.

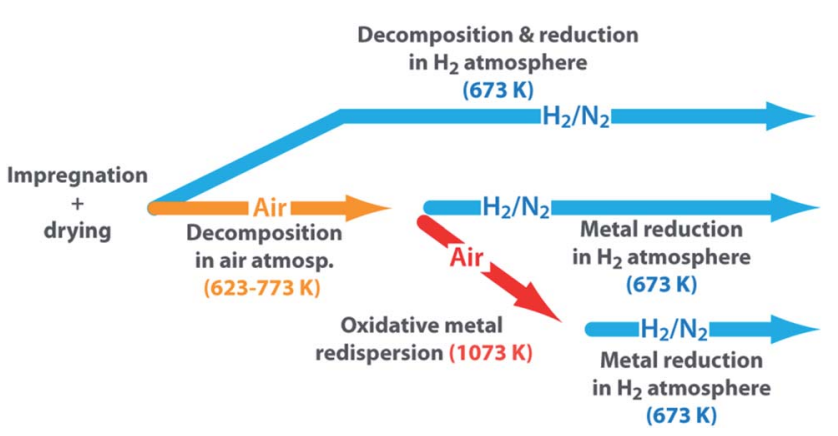

Fig. 1 Schematics of the different activation treatments applied from a single dry $\left(\mathrm{Pd}(\mathrm{acac})_{2}+\mathrm{Pt}(\mathrm{acac})_{2}\right) / \mathrm{MgO}$ impregnate to produce bimetallic catalysts. acac: acetyl acetonate. 


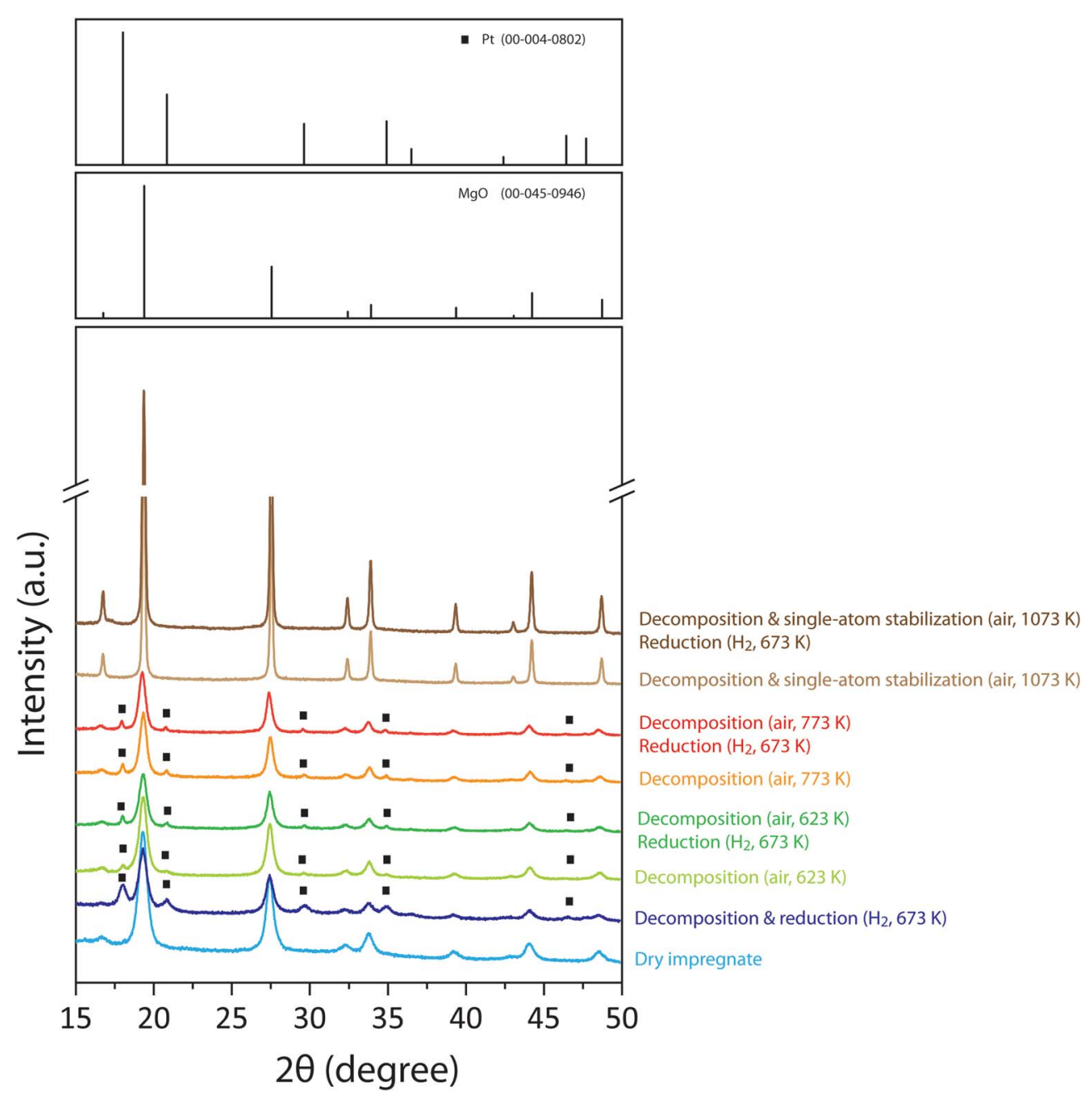

Fig. 2 X-ray diffraction patterns for PdPt/MgO materials synthesized from a common dry impregnate (bottom trace) following different activation routes. Selected diffraction patterns from the ICCD PDF-2 database are also included for reference.

Conventional activation routes based on air-calcination of the supported organometallic precursors, followed by a $\mathrm{H}_{2}$ reduction treatment, were also examined. Decomposition of the metal precursors in air at a temperature of either $633 \mathrm{~K}$ or $773 \mathrm{~K}$ resulted in weak, though comparatively sharper diffraction signals for Pt (Fig. 2). Again, no obvious signs for metallic Pd crystalline phases were detected by XRD. The observation of metallic species after calcination under an oxidative atmosphere indicates that the decomposition of the acetylacetonate moieties in the molecular precursors suffices to drive (partial) metal reduction to a zero-valent state. As judged from the narrower diffraction line-shape, larger Pt crystallites were obtained in this case compared to the direct thermal decomposition of the dry impregnate under a reducing $20 \% \mathrm{H}_{2} / \mathrm{N}_{2}$ atmosphere. Subsequent reduction led to a slight increase in relative intensity for the Pt diffractions, likely as a result of the expected increase in the degree of metal reduction. In marked contrast, annealing the air-calcined catalyst precursor to a higher temperature of $1073 \mathrm{~K}$ in air led, on the one hand, to a noticeable sharpening of the $\mathrm{MgO}$ diffractions indicating the sintering of the oxide support, but also to the complete vanishment of those diffractions corresponding to crystalline phases of the supported metals. This observation points to an oxidative disruption of metal(oxide) crystallites and redispersion of metallic phases into structures lacking long-range order. Interestingly, as shown in Fig. 2, neither after the subsequent $\mathrm{H}_{2}$-reduction treatment could diffraction signals for metallic phases be detected in this case, despite $\mathrm{H}_{2}$-temperature programmed reduction showing a noticeable cumulative $\mathrm{H}_{2}$ consumption of $0.08 \mathrm{mmol} \mathrm{H}_{2} \mathrm{~g}^{-1}$, associated to metal reduction, at temperatures $<673 \mathrm{~K}$ (Fig. S3†). In conjunction, these results suggest that, in contrast to any of the more conventional catalyst activation routes discussed above, oxidative metal redispersion results, after reduction, in metallic structures which are either amorphous in nature or highly dispersed, thus with very small, XRD-silent $(<2 \mathrm{~nm})$ crystalline domains.

In order to further explore the bounds of the crystallite oxidative redispersion phenomenon for the synthesis of PdPt/ $\mathrm{MgO}$ bimetallic catalysts, a set of materials with various surface-specific metal contents in the range of $0.2-4.0 \mathrm{M}_{\mathrm{at}} \mathrm{nm}^{-2}$ $(\mathrm{M}=\mathrm{Pd}+\mathrm{Pt}, \mathrm{Pd}: \mathrm{Pt}=1)$ were synthesized by impregnation with the acetyl acetonate metal precursors, followed by air 
calcination and further annealing at $1073 \mathrm{~K}$. The X-ray diffractograms for this series of materials, both after air-annealing and following the subsequent reduction treatment under hydrogen, are compiled in Fig. S4. $\dagger$ For reference purposes, also the diffractogram for the metal-free $\mathrm{MgO}$ carrier, submitted to the same air annealing treatment, is shown. Only diffractions corresponding to $\mathrm{MgO}$ are detected for catalysts with metal contents $\leq 1 \mathrm{M}_{\mathrm{at}} \mathrm{nm}^{-2}$ indicating that highly dispersed metal species, with crystalline domains below the detection limit of XRD are obtained in this range of metal contents. In contrast, further increasing metal loading to $\geq 2 \mathrm{M}_{\mathrm{at}} \mathrm{nm}^{-2}$ led to observable diffraction signals corresponding to PdO and fcc metallic crystals. In this case, the latter diffractions could be indexed to a PdPt alloy phase given the $d$-spacing correspondence with an equimolar, bulk PdPt alloy (Fig. S2 $\dagger$ ). Particularly the alloy phase grew in relative intensity upon increasing metal content from $2.0 \mathrm{M}_{\mathrm{at}} \mathrm{nm}^{-2}$ to $4.0 \mathrm{M}_{\mathrm{at}} \mathrm{nm}^{-2}$. Following the successive $\mathrm{H}_{2}$ reduction treatment, no signs of crystalline phases could be detected for catalysts with metal contents $\leq 1 \mathrm{M}_{\text {at }}$ $\mathrm{nm}^{-2}$. In the case of materials with metal loadings $\geq 2 \mathbf{M}_{\mathrm{at}}$ $\mathrm{nm}^{-2}$, those diffractions for PdO vanished completely, indicating the reduction of palladium oxide. Simultaneously, signals for PdPt grew in intensity, signifying further development of alloy crystals via reduction of metal oxidic species.

The results indicate that metal (oxide) crystallite disaggregation into highly dispersed species can be achieved via the high-temperature annealing $(1073 \mathrm{~K})$ in an air atmosphere, up to surface metal contents of $c a .1 \mathrm{M}_{\mathrm{at}} \mathrm{nm}^{-2}$. Surplus in metal content beyond this threshold lead to aggregation into metal(oxide) crystals. In the case of palladium, separate PdO crystals are clearly observed, whereas in the case of Pt only metallic phases are detected by XRD. This is in keeping with the known instability of platinum oxides at temperatures in excess to $c a$. $600 \mathrm{~K}$, which typically results in the release of lattice oxygen even under oxidative gas environments. ${ }^{47}$

Analysis of the evolution of the specific surface area after air annealing with the metal content provided further insights into the metal redispersion phenomenon. As shown in Fig. S5, $\uparrow$ the as-synthesized MgO support showed a BET specific surface area of $250 \mathrm{~m}^{2} \mathrm{~g}^{-1}$. Annealing to $1073 \mathrm{~K}$ led to a very significant sintering, reflected in a $c a$. seven-fold drop in surface area to 34 $\mathrm{m}^{2} \mathrm{~g}^{-1}$, indicating that the applied annealing temperature promotes surface mobility and agglomeration on $\mathrm{MgO}$. After metal deposition, however, increasingly higher specific surface areas in the range of $37-57 \mathrm{~m}^{2} \mathrm{~g}^{-1}$ were stabilized after annealing for $\mathrm{PdPt} / \mathrm{MgO}$ materials with increasing metal contents from 0.2 to $1 \mathrm{M}_{\mathrm{at}} \mathrm{nm}^{-2}$. This stabilization levels off at metal contents $\geq 1 \mathrm{M}_{\mathrm{at}} \mathrm{nm}^{-2}$. At even higher loadings $\left(>2 \mathrm{M}_{\mathrm{at}}\right.$ $\mathrm{nm}^{-2}$ ) the BET surface area decreases again. This behaviour is reminiscent of that observed previously for series of monometallic supported catalysts synthesized by dispersing different $4 \mathrm{~d}$ and $5 \mathrm{~d}$ transition metals on $\mathrm{CeO}_{2}$ via air-annealing at 1073 $\mathrm{K},{ }^{48}$ for which a comparable stabilization of the oxide carrier against sintering was observed also up to surface-specific metal contents of ca. $1 \mathrm{M}_{\mathrm{at}} \mathrm{nm}^{-2}$.

\section{Electron microscopy insights into metal dispersion}

Spherical aberration-corrected high-angle annular dark-field scanning-transmission electron microscopy (Cs-HAADF-STEM) was applied to study the dispersion of metal species at various stages throughout catalyst synthesis. Fig. 3 shows representative micrographs for $1.0 \mathrm{PdPt} / \mathrm{MgO}$ catalysts obtained via the activation of a common dry impregnate precursor through either decomposition of the metal precursors by air-calcination at $623 \mathrm{~K}$ or $773 \mathrm{~K}$, followed by reduction in $20 \% \mathrm{H}_{2} / \mathrm{N}_{2}$ at $673 \mathrm{~K}$, or via the combination of both decomposition and reduction steps in a single treatment under $20 \% \mathrm{H}_{2} / \mathrm{N}_{2}$ at $673 \mathrm{~K}$. As observed, in all cases, very small metal nanoparticles $(<5 \mathrm{~nm})$ coexist with larger metal agglomerates (up to $>50 \mathrm{~nm}$ in size). These results are in line with those retrieved with XRD, indicating that the latter population of large metal crystals are by and large responsible for the observed diffraction signals. Compositional analysis of the latter population by energydispersive X-ray spectroscopy (EDS) additionally revealed differences in composition among particles, with a significant fraction of the metal agglomerates being notably enriched in platinum with respect to the equimolar overall composition, indicating severe metal segregation. Both metal segregation and the markedly bimodal metal dispersion are undesired features for catalysis, given that a significant fraction of $\mathrm{Pt}$ remains unmixed and inaccessible to reactants, in the bulk of large crystals.

A notably different picture aroused when the catalyst precursor underwent air annealing at $1073 \mathrm{~K}$ following the air decomposition of the organometallic precursors. As shown in Fig. 4, no metal aggregates could be observed after airannealing, in contrast to any of the counterpart materials activated through more conventional procedures (Fig. 3). Highermagnification imaging revealed isolated metal atoms, which can be identified owing to their considerably higher Z-contrast, on the MgO surface (Fig. 4a and b). An appealing feature of HAADF-STEM is that the measured contrast intensity $(I)$ follows a simple relationship with the atomic number $(Z)$ for sufficiently thin samples, i.e. $I=k \cdot Z^{\alpha}$, where $k$ is a constant under a given set of experimental imaging conditions. The exponent $\alpha$ would take a value of 2 for a genuine Rutherford electron scattering phenomenon. ${ }^{49}$ In practice, values in the range of 1.7 \pm 0.3 are typically observed due to screening effects associated to atom core electrons. ${ }^{\mathbf{5 0 , 5 1}}$ Fig. $4 \mathrm{c}$ and $\mathrm{d}$ show the result of a local contrast analysis for those areas framed in Fig. 4a, each populated with five isolated metal atoms. In both areas, atomsized motifs can be grouped into two major levels of contrast intensity, which are marked with diamonds and stars, respectively, on the corresponding 3D contour plots. Analysis of the average intensity differences between these two levels, corrected for the contrast contribution from the $\mathrm{MgO}$ support, and considering the difference in atomic number between $\operatorname{Pd}(Z=$ 46) and $\operatorname{Pt}(Z=78)$, translates into an experimental value for the exponent $\alpha$ of 1.4 in the HAADF intensity relation, which falls well within the range of expected values. This analysis supports the assignment of the atom-sized motifs to Pd and Pt atoms, 


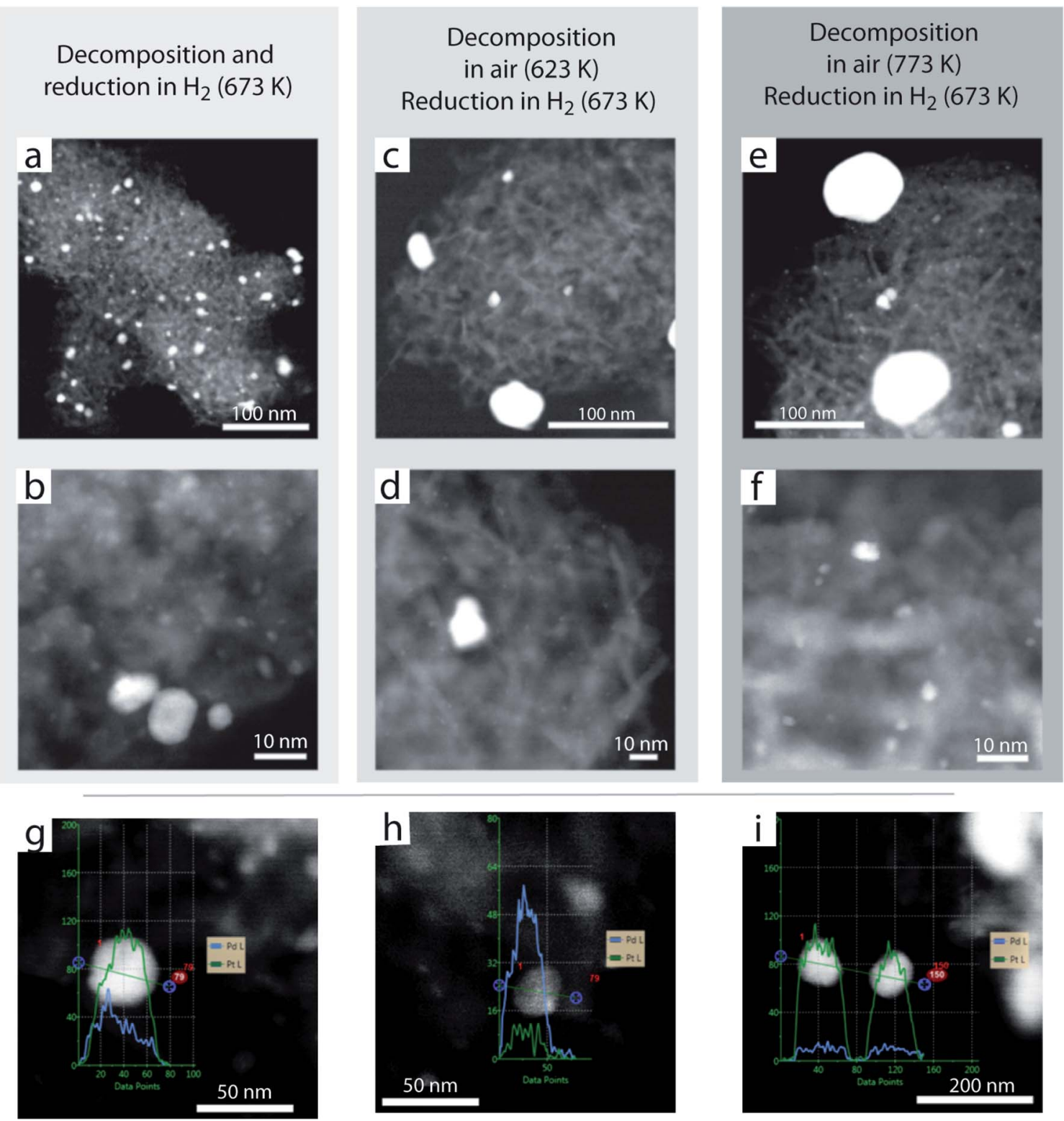

Fig. 3 HAADF-STEM micrographs of PdPt/MgO catalysts ( $\mathrm{Pd}: \mathrm{Pt}=1)$ obtained from an identical dry impregnate precursor via different precursor decomposition routes, i.e. by treating the dry impregnate directly in $20 \% \mathrm{H}_{2} / \mathrm{N}_{2}$ at $673 \mathrm{~K}(\mathrm{a}$, b); by decomposing the metal precursor in air at $623 \mathrm{~K}$ followed by metal reduction in $20 \% \mathrm{H}_{2} / \mathrm{N}_{2}$ at $673 \mathrm{~K}(\mathrm{c}$, d); and by decomposing the metal precursor in air at $623 \mathrm{~K}$, followed by air-calcination at $773 \mathrm{~K}$ and metal reduction in $20 \% \mathrm{H}_{2} / \mathrm{N}_{2}$ at $673 \mathrm{~K}(\mathrm{e}, \mathrm{f})$. Panels $\mathrm{g}-\mathrm{i}$ show close-up details, alongside line EDS compositional scans for representative large (>20 nm) metal nanoparticles in panel (a).

respectively, isolated, within nanometer distances, on the $\mathrm{MgO}$ surface.

Following the reduction treatment, the surface of the $\mathrm{MgO}$ crystals became populated with metal nanoclusters, as a result of the reductive agglomeration of the atomically dispersed species (Fig. 4e-h). In contrast to previously discussed catalyst activation procedures, a monomodal and remarkably narrow size distribution arises, which is centred at around $1.2 \mathrm{~nm}$ (Fig. 4h), i.e. corresponding to $c a$. 60 metal atoms per cluster. Moreover, as shown in Fig. 4f, EDS analysis revealed the bimetallic character of these clusters. The EDS linescans, collected while scanning the sample with the Cs-corrected e- beam probe ( $c a .0 .1 \mathrm{~nm}$ diameter), did not indicate any substantial metal segregation, or a core-shell metal distribution which have previously been computationally predicted for sub$\mathrm{nm}$ PdPt nanocluster models, ${ }^{52,53}$ suggesting mixing of the two metals directly after reduction activation. No larger metal agglomerates could be observed by STEM, which is in agreement with the absence of contributions from metallic crystals in the corresponding X-ray diffractograms, both after air annealing and following subsequent reduction. These results might now be further rationalized in view of the complementary electron microscopy insights. On the one hand, after hightemperature air annealing, metal species appear to be 
Prior to reduction
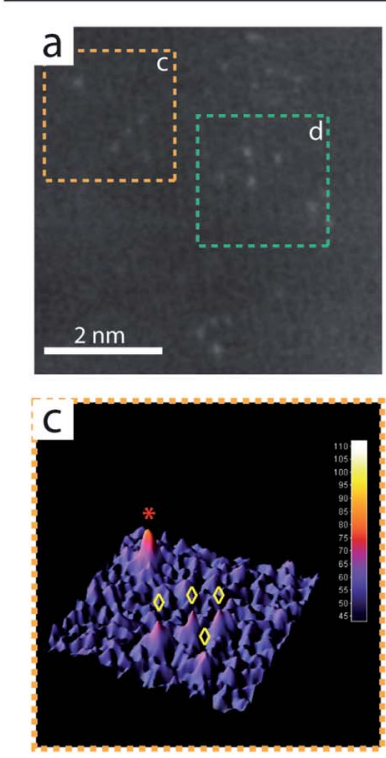
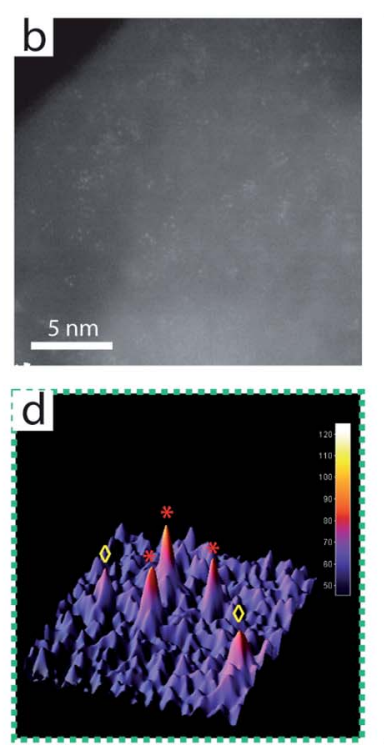

After reduction
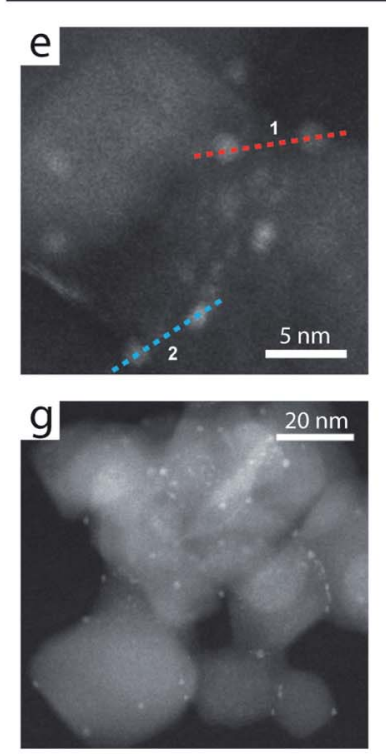

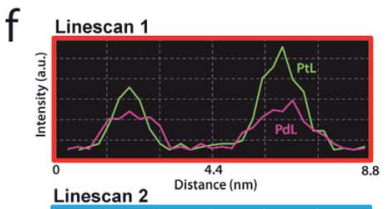

Linescan 2
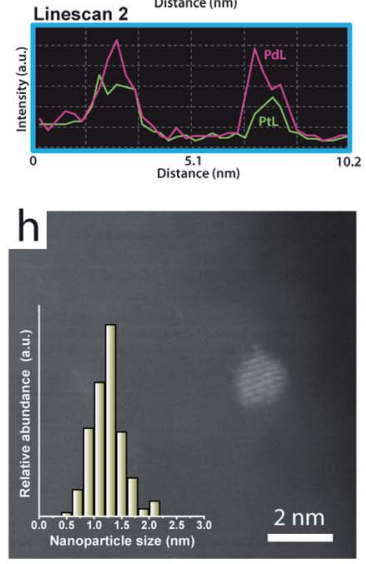

Fig. 4 HAADF-STEM analysis of 1.0PdPt/MgO after air calcination and annealing at $1073 \mathrm{~K}\left(\mathrm{a}-\mathrm{d}\right.$ ) and subsequent $\mathrm{H}_{2}$-reduction at $673 \mathrm{~K}$ (e-h). Panels (b) and (e) provide representative micrographs of the two materials at identical magnification, illustrating the reductive agglomeration of atomically dispersed metals into bimetallic nanoclusters following $\mathrm{H}_{2}$-reduction. Panel (a) shows a close-up view of a region in panel (b) where the isolated metal atoms can be discerned. Panels (c) and (d) display 3D Z-contrast maps of the corresponding regions framed in panel (a). Panel $f$ shows the EDS compositional profiles determined along the line scans depicted on panel e. The inset to panel (h) shows the nanoparticle size distribution determined after $\mathrm{H}_{2}$-reduction.

atomically trapped on $\mathrm{MgO}$, therefore lacking long range order. On the other hand, after $\mathrm{H}_{2}$-reduction, the very small size of those bimetallic aggregates produced via reductive agglomeration from the atomically dispersed precursor, falls below the minimum crystalline domain size detectable by XRD.

\section{In situ X-ray absorption spectroscopy}

X-ray absorption spectroscopy was applied to assess the dispersion, reduction kinetics and local coordination with metal specificity. Fig. 5 shows the extended X-ray absorption fine-structure (EXAFS) spectra recorded at the PdK and $\mathrm{PtL}_{3}$ absorption edges, respectively, for 1.0PdPt/MgO after oxidative metal redispersion at $1073 \mathrm{~K}$. The spectra showed no evidence for neither 1st-shell $\mathrm{M}-\mathrm{M}$ nor 2nd-shell $\mathrm{M}-\mathrm{O}-\mathrm{M}$ coordination, compatible with the absence of neither metallic nor metal oxide agglomerates. For both metals, the spectra could be fitted to a structural model where isolated metal atoms isomorphically substitute $\mathrm{Mg}^{2+}$ in direct sub-surface crystallographic positions underneath $\mathrm{MgO}$ step-edges, in agreement with previous results for monometallic catalysts synthesized similarly. ${ }^{54}$ Alternatively, structural models with metal cations sitting at more generic sub-surface $\mathrm{MgO}$ positions with a vicinal $\mathrm{V}_{\mathrm{Mg}}^{\prime \prime}$ vacancy, provide also an acceptable description of the experimental spectra (Fig. S6 $\dagger$ ). As reported recently by our group, ${ }^{54}$ alternative site models, such as those proposed earlier, based on metal atoms adsorbed onto F-centers or divacancy sites on the MgO surface, ${ }^{55,56}$ with lower first- and second-shell coordination numbers for the metal adatom, did not provide a sensible fit of the experimental EXAFS data. These results indicate that both metals become atomically dispersed and stabilized by the $\mathrm{MgO}$ lattice following the high temperature oxidative redispersion treatment.

The reduction behaviour of the atomically dispersed bimetallic 1.0PdPt/MgO was studied with in situ X-ray absorption near-edge structure (XANES) spectroscopy. Fig. 6 shows the temperature-resolved XANES spectra recorded at the PdK and $\mathrm{PtL}_{3}$ edges, respectively, during the in situ reduction of the catalyst under flow of $20 \% \mathrm{H}_{2} / \mathrm{N}_{2}$ in the temperature range of 298-773 K. For comparison, results for monometallic 0.5Pd/ $\mathrm{MgO}$ and $0.5 \mathrm{Pt} / \mathrm{MgO}$ analogues, synthesized identically, are also included. In both cases, progressive dampening of the white line intensity indicated metal reduction. Metal-specific information on the reduction kinetics was extracted via a MCR-ALS chemometric method to track the evolution of the concentration of the oxidized and reduced components as a function of the temperature. As shown in Fig. 6c and f, a decline of the oxidic contribution to the convoluted spectra sets in from milder temperatures for Pd, and drops faster as the temperature increases in the range $<523 \mathrm{~K}$, indicating that $\mathrm{Pd}$ reduction proceeds at lower temperatures than that of Pt. Moreover, comparison of the concentration profiles determined by MCR-ALS for mono- and bimetallic counterparts reveals that the concomitant presence of both metals on MgO shifts Pt reduction to lower temperatures, suggesting that hydrogen activation and spillover from nascent Pd clusters assists the reduction of more stable oxidic Pt species.

Following in situ reduction in the XAS cell, EXAFS spectra were recorded to investigate the average local coordination of 
a)

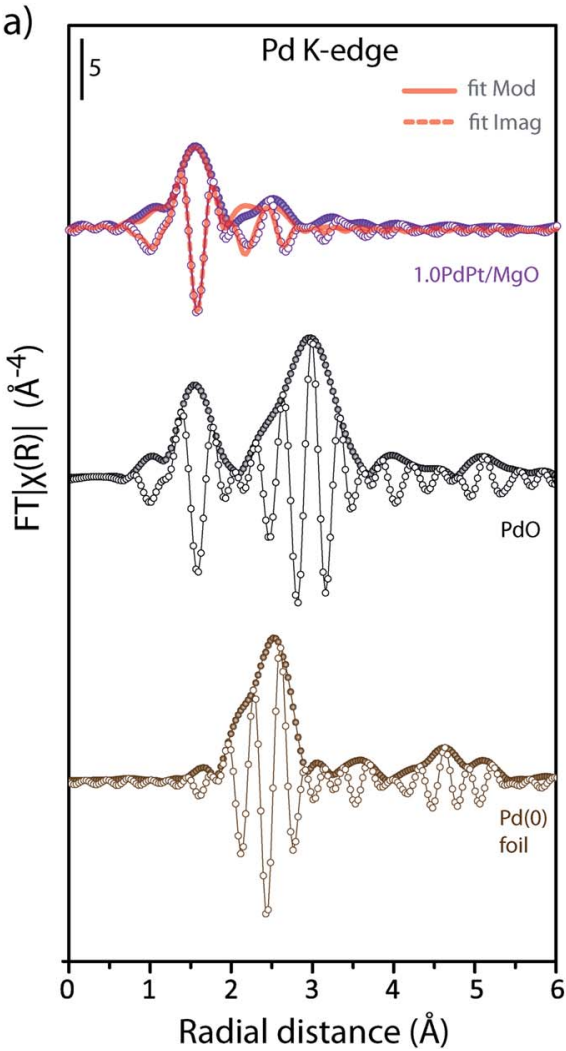

b)

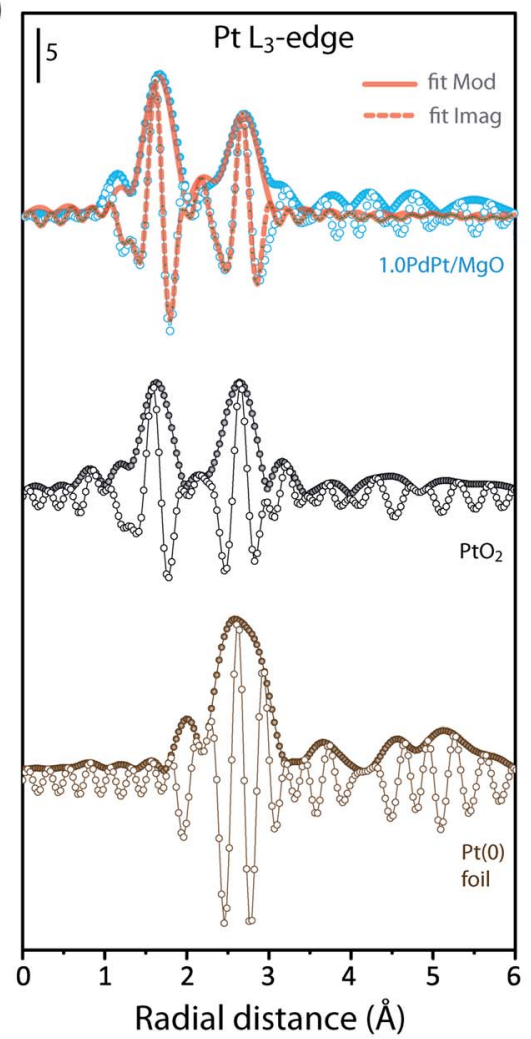

c)
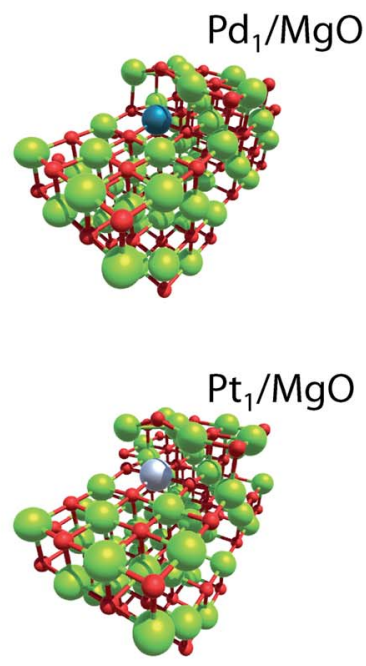

Fig. 5 X-ray absorption spectroscopy characterization of atomically dispersed bimetallic PdPt/MgO catalysts. (a-b) Fourier-transform of the phase-uncorrected $k^{3}$-weighted EXAFS function for spectra recorded at the $\mathrm{Pd}-\mathrm{K}(\mathrm{a})$ and $\mathrm{Pt}-\mathrm{L}_{3}$ (b) XAS edges for $1.0 \mathrm{PdPt} / \mathrm{MgO}$ synthesized via oxidative metal redispersion at $1073 \mathrm{~K}$. The corresponding spectra for bulk metal oxides and metallic foils reference materials are also included. Scatter traces correspond to experimental data (closed symbols: real components; open symbols: imaginary components). Full lines correspond to the fits of the experimental data to structural models corresponding to single metal atoms sitting under a MgO step-edge, depicted on panel (c). See Tables S2 and S3 in the ESI $\uparrow$ for fitting results.

the two metals. Fig. 7 shows the corresponding spectra for $1.0 \mathrm{PdPt} / \mathrm{MgO}$ after reduction of the atomically dispersed bimetallic precursor. Spectra for the oxidic precursor, metallic foils and the corresponding atomically dispersed monometallic $0.5 \mathrm{Pd} / \mathrm{MgO}$ and $0.5 \mathrm{Pt} / \mathrm{MgO}$ after reduction, are also shown for comparison. Wavelet transforms of the PdK-EXAFS spectra for the $e x$-single-atom 1.0PdPt/MgO after reduction show essentially only $\mathrm{Pd}-\mathrm{M}(\mathrm{M}=\mathrm{Pd}, \mathrm{Pt})$ scattering contributions, indicating the quantitative reductive agglomeration of palladium. At the $\mathrm{PtL}_{3}$ edge, however, next to the 1st-shell metal-metal component, a second scattering contribution for $\mathrm{Pt}-\mathrm{O}$ is observed, indicating that a fraction of the atomically dispersed platinum cations, likely those stabilized in higher-confinement sub-surface positions, did not undergo reduction at the applied activation temperature and thus did not engage in metal agglomeration. This is in contrast to the results obtained for the corresponding 1.0PdPt/MgO bimetallic catalyst which had not undergone oxidative redispersion, for which no significant $\mathrm{M}-\mathrm{O}$ scattering contributions for either metal were observed following reduction under identical conditions (Fig. S7†), indicating that the lower metal reducibility in the former is associated to the oxidative redispersion of metal crystallites and the trapping and stabilization of isolated metal cations by the lattice of the MgO support.
Owing to the incomplete reduction of Pt species, and thus the convolution of different 1st- and 2nd-shell contributions to the corresponding $\mathrm{PtL}_{3}$-EXAFS spectra, further analyses on the bimetallic character of the resulting metal nanoclusters focused on spectral features at the PdK edge. After reduction activation, all FT EXAFS spectra show a scattering contribution centred at a radial distance of $2.5 \AA$ (without phase correction), corresponding to $\mathrm{M}-\mathrm{M}$ coordination in metallic clusters (Fig. 7a). Careful evaluation of the imaginary part (Fig. 7b), however, reveals significant differences in the relative intensity for those three function maxima in the radial distance region of 2.0-3.0 between mono- and bimetallic catalysts produced by reductive activation of atomically dispersed precursors. Whilst for $0.5 \mathrm{Pd} /$ $\mathrm{MgO}$, the amplitude of the three maxima increases progressively on increasing radial distance, for $1.0 \mathrm{PdPt} / \mathrm{MgO}$ the amplitude levelled off after the second maximum. The corresponding PdK-EXAFS spectra were simulated for Pd-Pd and PdPt paths in mono- and bimetallic clusters, respectively (see inset to Fig. 7b). For the Pd-Pd a systematic increase in the amplitude for the three maxima in the mentioned radial distance range of the imaginary FT component is observed, well in line with the experimental results for the monometallic $0.5 \mathrm{Pd} / \mathrm{MgO}$ catalyst. In contrast, the amplitude order reversed for the second and third maxima for the simulated spectra for the Pd-Pt path. 
a)

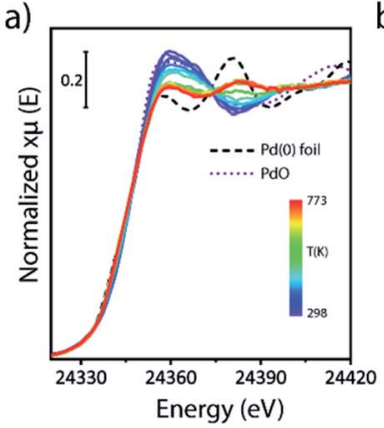

d)

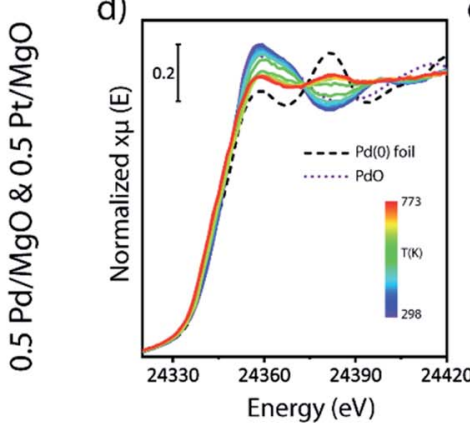

b)

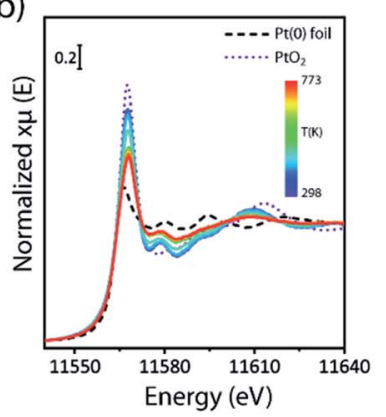

e)

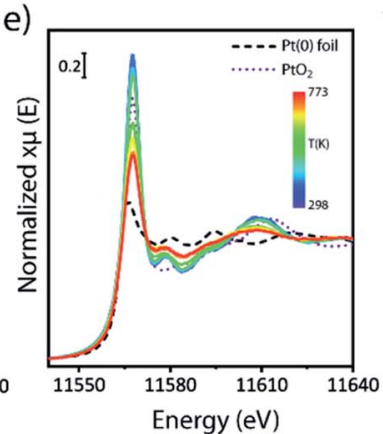

c)

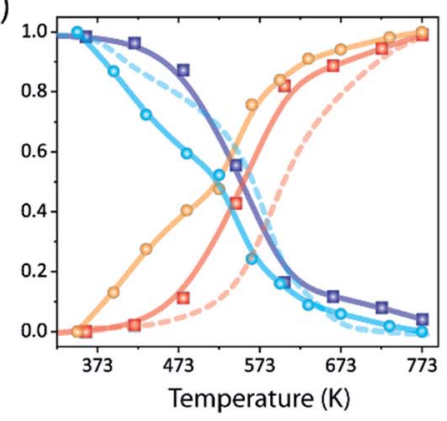

f)

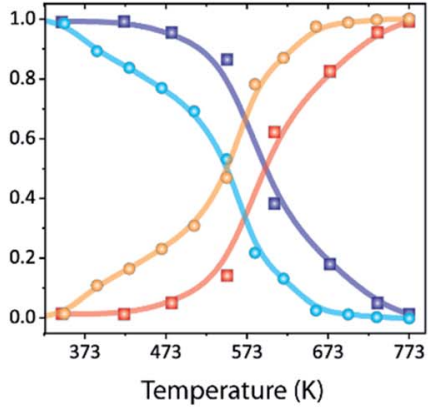

- Pd(K) oxidic component

- $\mathrm{Pd}(\mathrm{K})$ as-reduced component

a Pt(LIII) oxidic component

a $\mathrm{Pt}$ (LIII) as-reduced component

Fig. 6 Temperature-resolved X-ray Absorption Near-Edge Structure (XANES) analysis of metal reduction in a bimetallic 1.0PdPt/MgO catalyst $(\mathrm{a}-\mathrm{c})$ and the corresponding monometallic $0.5 \mathrm{Pd} / \mathrm{MgO}$ and $0.5 \mathrm{Pt} / \mathrm{MgO}$ monometallic counterparts (d-f). Temperature-resolved XANES spectra at the PdK-edge $(a, d)$ and the $\mathrm{PtL}_{3}$-edge $(b, e)$ during the exposure of the atomically dispersed catalyst precursors to a flow of $20 \% \mathrm{H}_{2} / \mathrm{N}_{2}$ in the temperature range of 298-773 K. The panels include the corresponding XANES spectra for reference metal foils and metal oxides $\left(\mathrm{PdO}\right.$ and $\mathrm{PtO} \mathrm{O}_{2}$ respectively). Temperature-resolved evolution of the relative abundance of the oxidic metal state and the as-reduced metal states in the temperature range screened. For the sake of direct comparison, dotted lines in panel (c) show the reduction behaviour for Pd and Pt, respectively, in the corresponding monometallic materials.

Moreover, the signals of the simulated EXAFS patterns are partially in anti-phase. These observations suggest strongly that the change in the monotony for the EXAFS FT-imaginary component for the bimetallic 1.0PdPt/MgO, compared to the monometallic $0.5 \mathrm{Pd} / \mathrm{MgO}$ counterpart, arises from a convolution of two contributions associated to Pd-Pd and Pd-Pt scattering paths in the former. These results tie in well with those from EDS nanospectroscopy and indicate the bimetallic nature of the small clusters developed on $\mathrm{MgO}$ upon reductive agglomeration from the atomically dispersed precursors. Fitting of the EXAFS spectra (Fig. S8 $\dagger$ ) resulted in average Pd-Pd and Pd-Pt coordination numbers (CN) of $2.5 \pm 0.8$ and $2.1 \pm$ 0.9 , indicative for the average very small size of the bimetallic nanoclusters (Table S4 $\dagger$ ). Taken together, XAS results demonstrate the atomically dispersed state of the catalyst precursors after the oxidative crystal redispersion treatment, the existence of bimetallic effects on the metal reduction kinetics and the development of bimetallic clusters following reductive agglomeration, with sizes at the lowest frontier of the nanometer regime.

\section{Catalysis}

To assess the implications of the thermal history-dependent metal aggregate size on catalytic applications under severe reaction conditions, $1.0 \mathrm{PtPd} / \mathrm{MgO}$ catalysts were evaluated in the dry reforming of methane (DRM) with $\mathrm{CO}_{2}$ at $923 \mathrm{~K}$. DRM is a reaction of eminent interest in the context of the valorisation of abundant (unconventional) natural gas resources with a simultaneous chemical recycling of waste $\mathrm{CO}_{2}$. Bimetallic nanoparticles, ${ }^{57-60}$ including PdPt, ${ }^{57}$ have shown significant potential with high reaction rates and limited carbon build-up at moderate temperatures. Catalysts synthesized by either air calcination at $623 \mathrm{~K}$ or oxidative redispersion at $1073 \mathrm{~K}$, followed, in both cases, by reduction at $673 \mathrm{~K}$ with hydrogen, were selected for the catalysis investigation. Reaction settings were adjusted to achieve approach-to-equilibrium factors $<0.01$ for this reversible reaction, ${ }^{46}$ thus ensuring kinetic control.

Under the applied reaction conditions, the neat $\mathrm{MgO}$ support showed a mass-specific methane conversion rate between sevenfold and more than one order of magnitude lower than any of the $\mathrm{PdPt} / \mathrm{MgO}$ catalysts, evidencing that the magnesia support and the reactor walls are rather inert at the applied process conditions and thus the supported metal clusters/nanoparticles account for the activity observed. With the bimetallic catalysts, in both cases, a transient reaction regime was observed for times on stream $<30 \mathrm{~h}$, during which the $\mathrm{CH}_{4}$ conversion rate increased (Fig. S9†). Similar "induction" regimes have been previously observed for bimetallic catalysts exposed to methane reforming conditions and have been ascribed to an initial reduction/surface restructuring of the active metallic phases. ${ }^{61}$ Following this transient period, the $e x$-single-atom $\mathrm{PdPt} / \mathrm{MgO}$ catalyst afforded a more than 

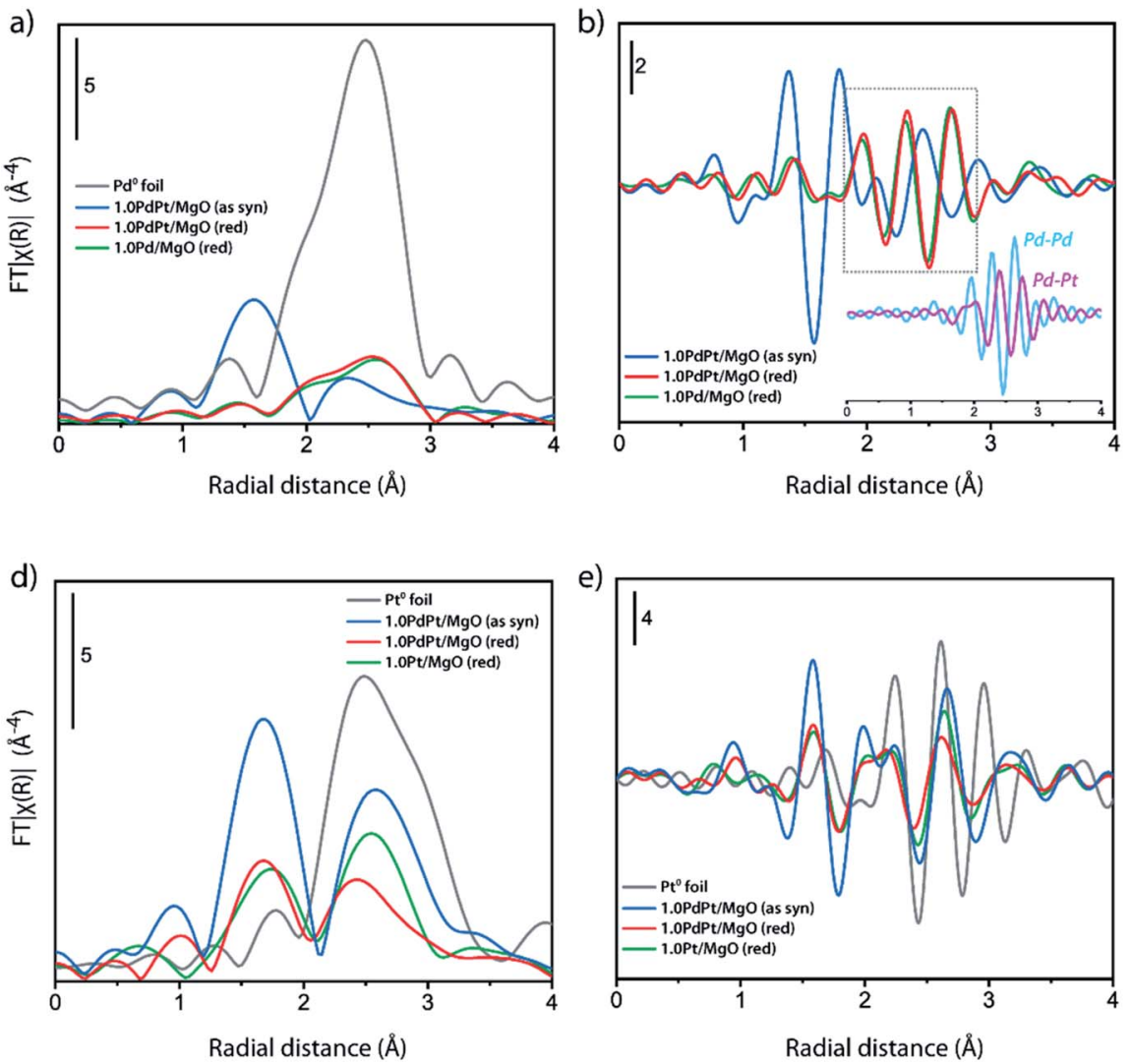

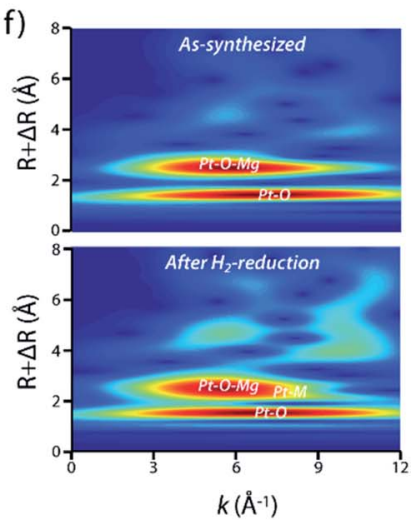

Fig. 7 X-ray absorption study of the structure of bimetallic clusters in PdPt/MgO. (a, d) Modulus and (b, e) imaginary Fourier-Transform (FT) terms of the EXAFS function, and wavelet transform of the EXAFS function $(c, f)$ at the $\mathrm{Pd}-\mathrm{K}(\mathrm{a}-\mathrm{c})$ and $\mathrm{Pt}-\mathrm{L}_{3}(\mathrm{~d}-\mathrm{f})$ absorption edges for a $1.0 \mathrm{PdPt} /$ $\mathrm{MgO}$ single-atom precursor, as-synthesized via oxidative redispersion at $1073 \mathrm{~K}$, and the corresponding catalyst after in situ reduction in $20 \% \mathrm{H}_{2} /$ $\mathrm{N}_{2}$. In panels ( $\mathrm{a}$ and $\mathrm{b}$ ) and ( $\mathrm{d}$ and $\mathrm{e}$ ), the corresponding spectra for monometallic $0.5 \mathrm{Pd} / \mathrm{MgO}$ and $0.5 \mathrm{Pt} / \mathrm{MgO}$ single-atom analogues in their oxidic state as well as the corresponding metallic foils are also included for reference purposes. The inset to panel (b) shows the imaginary FT component of simulated EXAFS spectra for a pure Pd crystal as well as a PdPt equimolar alloy. In panels (c) and (f), M stands for either Pd or Pt.

threefold higher conversion TOF (Fig. 8a). This observation agrees with the higher abundance of coordinatively unsaturated surface sites (cus) on the small bimetallic clusters developed on the latter catalyst, which are known to be more effective for the kinetically relevant $\mathrm{C}-\mathrm{H}$ bond cleavage in methane reforming catalysis. $^{\mathbf{4 6 , 6 2 , 6 3}}$ The metal-specific reaction rate achieved with the bimetallic clusters $\left(9.7 \times 10^{-2} \mathrm{~mol} \mathrm{~min}^{-1} \mathrm{~g}_{\text {metal }}{ }^{-1}\right)$ is higher than those reported for Ni-based catalysts at higher reaction temperatures in the range of 1023-1073 K. ${ }^{61,64} \mathrm{~A} \mathrm{H}_{2} / \mathrm{CO}$ molar ratio of 0.65 was determined for the syngas product following the transient period, which is slightly higher than the value of 0.55 predicted under the assumption of thermodynamic equilibrium considering the occurrence of both DRM and the reverse water-gas-shift (RWGS) reactions at the applied operation temperature.

Next to activity, analysis of the reaction performance for $c a$. $40 \mathrm{~h}$ on-stream, following the initial induction period, revealed remarkable differences in stability. Fig. 8b depicts the evolution of the normalized activity factor with the reaction time. No relevant decay in activity was observed for the most active 1.0PdPt/MgO catalyst generated from the corresponding atomically dispersed precursor. In marked contrast, a significant activity decay, corresponding to a zero-order deactivation rate constant of $3.6 \times 10^{-3} \mathrm{~h}^{-1}$, was obvious for the catalyst generated from metal oxide agglomerates on the $\mathrm{MgO}$ carrier. To shed light into these different stabilities under reaction conditions, HAADF-STEM was applied to study metal dispersion on the catalysts recovered after the catalytic test. As shown in Fig. 8c, the (sub)nanometer metal clusters were found to persist on the surface of the catalyst developed from an atomically dispersed precursor, where only few slightly larger, albeit $<5 \mathrm{~nm}$, metal nanoparticles could be detected. In contrast, the catalyst for which activation did not involve oxidative atomic redispersion, and thus it displayed a bimodal starting metal particle size distribution prior to catalysis, showed an evident depletion in the population of the smallest metal clusters after the prolonged exposure to reaction conditions. Essentially only those comparatively larger ( $>5 \mathrm{~nm}$ ) particles could be observed after catalysis. These results point to a significant metal particle growth in this case, which accounts for the observed catalyst deactivation. Reinforcing these microscopy observations, XRD provided also evidence for the limited growth experienced by the small metal clusters derived from single-atom precursors during exposure to reaction conditions (Fig. S10†). These results are reminiscent of the high stability observed previously with $\mathrm{Ni}-\mathrm{MgO}$ catalysts derived from solid solutions, ${ }^{\mathbf{6 1 , 6 4 , 6 5}}$ where the 
a)

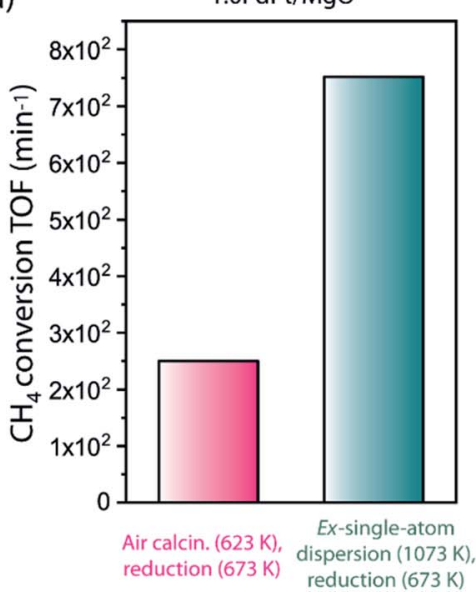

b)

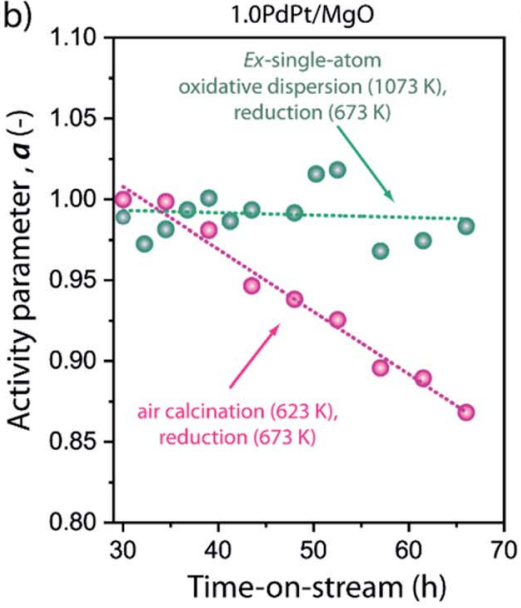

c)
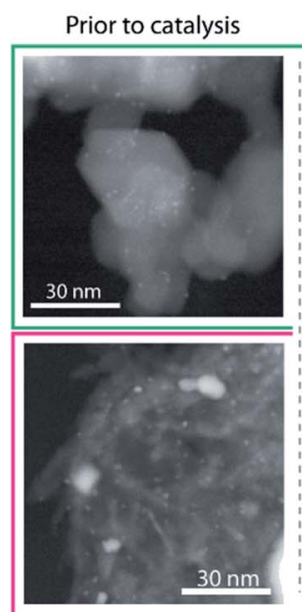

After catalysis
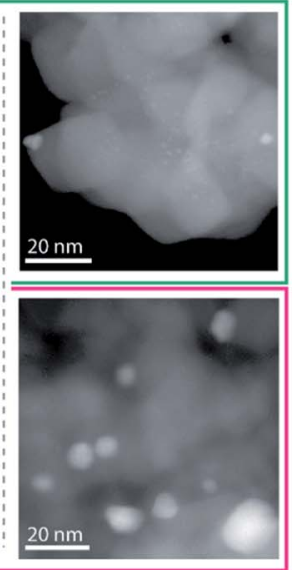

Fig. 8 Catalytic results for methane conversion on bimetallic PdPt/MgO catalysts. (a) Forward methane conversion rate per unit metal surface atom (Turnover Frequency, TOF); (b) evolution of the normalized activity parameter, i.e. $a=r_{\mathrm{CH}_{4}}(t) / \mathrm{CH}_{4}\left(t_{0}\right)$ with the time-on-stream, following the initial induction period observed after the initiation of the catalytic test ( $t_{0}=30 \mathrm{~h}$ ); and (c) representative HAADF-STEM micrographs prior to catalysis and after $\mathrm{ca} .70$ hours on stream under methane dry reforming conditions, for $1.0 \mathrm{PdPt} / \mathrm{MgO}$ catalysts activated from the same dry impregnate precursor via either air-calcination at $623 \mathrm{~K}$ or the same followed by air-annealing at $1073 \mathrm{~K}$ (oxidative metal redispersion into singleatom species), followed by reduction in $20 \% \mathrm{H}_{2} / \mathrm{Ar}$ at $673 \mathrm{~K}$. Reaction conditions: $T=923 \mathrm{~K}, \mathrm{P}=1 \mathrm{bar}$, feed $\mathrm{Ar} / \mathrm{CH}_{4} / \mathrm{CO}_{2}=4.4 / 1.5 / 1.0$ (molar), WHSV $=6.9 \mathrm{~h}^{-1}$.

active metal exsolves from lattice sites within the support under reaction conditions. It can therefore be inferred from these results that the narrow nanocluster size distribution achieved via reductive agglomeration from isolated metal atoms dampens metal growth processes even under high-temperature reaction conditions, in spite of their higher surface-to-volume ratio and thus intrinsic lower stability. This effect is thus likely associated to a minimization of the driving force for
Ostwald ripening mechanisms by the achievement of lowpolydispersity nanoclusters. On the contrary, such ripening processes are favoured with the markedly bimodal metal nanoparticle size distributions obtained via more conventional catalyst synthesis and activation protocols.

Jointly, the results underscore how adjusting both the surface metal content and the thermal activation route is essential for the development and stabilization of very small

$$
\begin{array}{ll}
0 & 0 \\
0 & \mathrm{Mg} \\
0 & \mathrm{Pd} \\
0 & \mathrm{Pt} \\
0 & \text { lacac] }^{2}
\end{array}
$$

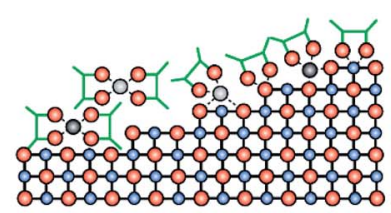

Decomposition \& reduction in $\mathrm{H}_{2}$ atmosphere $(673 \mathrm{~K})$

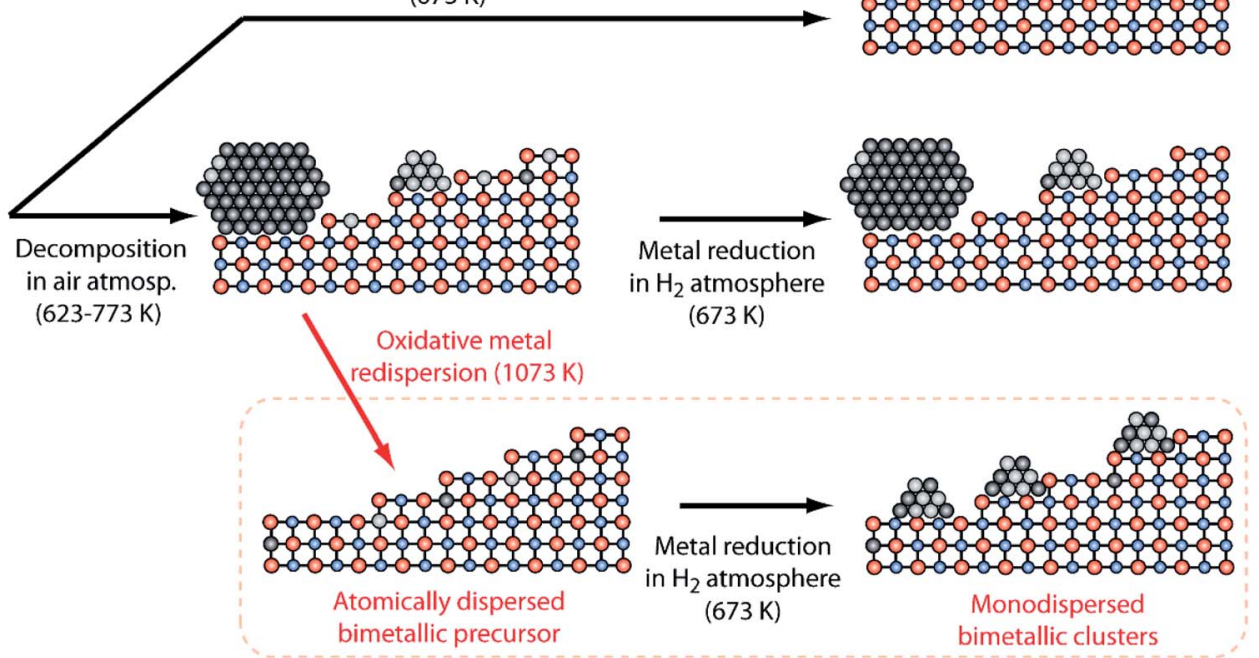

Fig. 9 Schematic representation of the nanostructure of $\mathrm{PdPt} / \mathrm{MgO}$ materials obtained from a common dry impregnate via the decomposition of metal acetylacetonate precursors through various activation procedures. The route in the dotted frame corresponds to the oxidative crystal redispersion and atom trapping into atomically dispersed metals on $\mathrm{MgO}$, followed by reductive agglomeration into $\sim 1 \mathrm{~nm}$ monodispersed bimetallic clusters. 
supported bimetallic nanoparticles. Fig. 9 summarizes graphically the different explored paths from a common starting dry impregnate and the consequences for metal mixing and dispersion. Following the simultaneous deposition of acetyl acetonate compounds of the two selected metals on the $\mathrm{MgO}$ support, direct decomposition of these metal precursors and metal reduction to zero-valent state under a reductive atmosphere $\left(20 \% \mathrm{H}_{2} / \mathrm{N}_{2}\right)$ leads to a markedly bimodal metal particle size distribution, combining very small $(<3 \mathrm{~nm})$ nanoparticles with larger $(>10 \mathrm{~nm})$ agglomerates, as well as a noticeable sizedependent metal segregation, and thus suboptimal metal mixing. Decomposition of the molecular metal precursors via air calcination (623-773 K) results in highly dispersed metal oxide species, however, accompanied by bulkier metallic agglomerates. Further thermal treatment in the presence of $\mathrm{H}_{2}$ leads to an essentially complete metal reduction which, however, does not revert the bimodal size distribution and metal segregation occurred during air calcination. However, when higher temperature air annealing follows decomposition of the metal precursors, instability of metal (oxide) crystals drives oxidative disruption into mobile monoatomic species of each metal which, up to an overall surface content of $c a .1 \mathrm{M}_{\mathrm{at}} \mathrm{nm}^{-2}$, can be trapped and stabilized by the oxide carrier at near-surface lattice positions. Further reductive metal agglomeration from isolated (single) atoms of the two metals, initially spaced within nanometer distances, leads to the development of uniformly sized, down to sub-nanometric, bimetallic clusters with particularly high stability to sintering even under harsh reaction conditions. While this synthesis concept is illustrated in greater detail for the showcase of PdPt nanoclusters, the methodology holds broader generality. Indeed, an identical synthesis route has been demonstrated to also develop (sub)nanometer PtIr bimetallic clusters on MgO (Fig. S11 in the ESI $\dagger$ ).

\section{Conclusions}

The combination of oxidative disruption of metal (oxide) crystallites at high temperatures with atom-trapping phenomena on nanoparticulate oxides enables single-atom species for two different metals to be stabilized, within nanometer distances, on a common oxide support material. For supported bimetallic $\mathrm{PdPt} / \mathrm{MgO}$ catalysts, atomic resolution HAADF-STEM, X-ray diffraction and X-ray absorption spectroscopy show that this atomic co-dispersion for the two metals is effective up to an overall surface-specific metal content of about $1 \mathrm{M}_{\mathrm{at}} \mathrm{nm}^{-2}$, beyond which the metal atom-trapping capacity of the $\mathrm{MgO}$ support appears to exhaust and metal clustering into crystallites sets in. Subsequent reductive metal agglomeration using hydrogen as reducing agent shows the reduction kinetics to differ for each metal, and reveals bimetallic effects, likely due to $\mathrm{H}_{2}$ dissociation and spillover effects from early nascent metallic nanoclusters. Unlike more conventional activation routes based on air-calcination of the metal precursors at milder temperatures $(623-773 \mathrm{~K})$ followed by reduction, which result in bimodal metal particle size distributions and noticeable metal segregation, the reductive activation from single-atom dispersed bimetallic precursors delivers very small $(c a .1 \mathrm{~nm})$ and bimetallic clusters on the oxide carrier. The latter display higher catalytic activity and a remarkable stability under relevant conditions for the high-temperature (923 K) dry reforming of methane with $\mathrm{CO}_{2}$ into syngas, likely as a result of a minimized driving force for Ostwald ripening particle growth mechanisms associated to the high cluster monodispersity. The synthesis route, which is herein shown to be applicable to different combinations of $4 \mathrm{~d}$ and $5 \mathrm{~d}$ metals, provides access to very small, (sub)nanometer sized bimetallic clusters with high stability for applications in catalysis and other technologies where combining bimetallic effects, highly reactive metal surfaces and high thermal stability is important.

\section{Conflicts of interest}

There are no conflicts to declare.

\section{Acknowledgements}

XAS experiments were performed at the CLAESS BL22 beamline of the ALBA Light Source, Cerdanyola del Vallés, Spain: experiment 2019023278. The authors are grateful to beamline staff members, as well as to E. Andrés, M. E. Martínez-Monje and I. López-Luque (ITQ) for assistance with XAS measurements. J. Ternieden (MPI-KOFO) is acknowledged for XRD measurements. M. Mayer (MPI-KOFO) is thanked for his assistance with the synthesis of the MgO support. P. N. Plessow and F. Studt (KIT) are gratefully acknowledged for the development of the DFT-optimized structural models used in EXAFS data fitting. Authors are grateful to Prof. Ferdi Schüth for providing lab facilities and supportive access to analytical methods at the MPI-KOFO. This research received funding from the Max Planck Society and the Spanish Ministry of Science and Innovation (RTI2018-096399-A-100 and SEV-2016-0683). B. B. S. acknowledges the Alexander von Humboldt Foundation for a postdoctoral grant. M. G. F. acknowledges the Spanish Ministry of Science, Innovation and Universities for a FPU predoctoral scholarship (FPU17/04701). Open Access funding provided by the Max Planck Society.

\section{References}

1 M. Zhou, C. Li and J. Fang, Chem. Rev., 2021, 121, 736-795. 2 J. Rick, M.-C. Tsai and B. Hwang, Nanomaterials, 2015, 6, 5.

3 Q. Shao, P. Wang and X. Huang, Adv. Funct. Mater., 2019, 29, 1806419.

4 B.-W. Zhang, H.-L. Yang, Y.-X. Wang, S.-X. Dou and H.-K. Liu, Adv. Energy Mater., 2018, 8, 1703597.

5 D. M. Alonso, S. G. Wettstein and J. A. Dumesic, Chem. Soc. Rev., 2012, 41, 8075.

6 K. Loza, M. Heggen and M. Epple, Adv. Funct. Mater., 2020, 30, 1909260.

7 M. Sankar, N. Dimitratos, P. J. Miedziak, P. P. Wells, C. J. Kiely and G. J. Hutchings, Chem. Soc. Rev., 2012, 41, 8099.

8 K. D. Gilroy, A. Ruditskiy, H.-C. Peng, D. Qin and Y. Xia, Chem. Rev., 2016, 116, 10414-10472. 
9 R. Ferrando, J. Jellinek and R. L. Johnston, Chem. Rev., 2008, 108, 845-910.

10 X. Peng, Q. Pan and G. L. Rempel, Chem. Soc. Rev., 2008, 37, 1619-1628.

11 J. H. Sinfelt, J. L. Carter and D. J. C. Yates, J. Catal., 1972, 24, 283-296.

12 J. Schwank, AIChE J., 1985, 31, 1405.

13 S. I. Murahashi, T. Naota and N. Hirai, J. Org. Chem., 1993, 58, 7318-7319.

14 D. I. Enache, J. K. Edwards, P. Landon, B. Solsona-Espriu, A. F. Carley, A. A. Herzing, M. Watanabe, C. J. Kiely, D. W. Knight and G. J. Hutchings, Science, 2006, 311, 362365.

15 N. Agarwal, S. J. Freakley, R. U. McVicker, S. M. Althahban, N. Dimitratos, Q. He, D. J. Morgan, R. L. Jenkins, D. J. Willock, S. H. Taylor, C. J. Kiely and G. J. Hutchings, Science, 2017, 358, 223-227.

16 M. Zaarour, J. Cazemier and J. Ruiz-Martínez, Catal. Sci. Technol., 2020, 10, 8140-8172.

17 P. Lu, T. Teranishi, K. Asakura, M. Miyake and N. Toshima, J. Phys. Chem. B, 1999, 103, 9673-9682.

18 S. Hermans, R. Raja, J. M. Thomas, B. F. G. Johnson, G. Sankar and D. Gleeson, Angew. Chem., Int. Ed., 2001, 40, 1211-1215.

19 J. K. Edwards, B. Solsona, E. Ntainjua N, A. F. Carley, A. A. Herzing, C. J. Kiely and G. J. Hutchings, Science, 2009, 323, 1037-1041.

20 F. Studt, F. Abild-Pedersen, T. Bligaard, R. Z. Sorensen, C. H. Christensen and J. K. Norskov, Science, 2008, 320, 1320-1322.

21 J. M. Parera and J. N. Beltramini, J. Catal., 1988, 112, 357365.

22 H. Miura, Y. Tanaka, K. Nakahara, Y. Hachiya, K. Endo and T. Shishido, Angew. Chem., Int. Ed., 2018, 57, 6136-6140.

23 M. Chen, D. Kumar, C. W. Yi and D. W. Goodman, Science, 2005, 310, 291-293.

24 F. Gao and D. W. Goodman, Chem. Soc. Rev., 2012, 41, 8009. 25 F. (Feng) Tao, Chem. Soc. Rev., 2012, 41, 7977.

26 N. Toshima and T. Yonezawa, New J. Chem., 1998, 22, 11791201.

27 P.-C. Chen, X. Liu, J. L. Hedrick, Z. Xie, S. Wang, Q.-Y. Lin, M. C. Hersam, V. P. Dravid and C. A. Mirkin, Science, 2016, 352, 1565-1569.

28 O. S. Alexeev and B. C. Gates, Ind. Eng. Chem. Res., 2003, 42, 1571-1587.

29 A. Wong, Q. Liu, S. Griffin, A. Nicholls and J. R. Regalbuto, Science, 2017, 358, 1427-1430.

30 K. Ding, D. A. Cullen, L. Zhang, Z. Cao, A. D. Roy, I. N. Ivanov and D. Cao, Science, 2018, 362, 560-564.

31 M. Treguer, C. de Cointet, H. Remita, J. Khatouri, M. Mostafavi, J. Amblard, J. Belloni and R. de Keyzer, J. Phys. Chem. B, 1998, 102, 4310-4321.

32 Y. Mizukoshi, K. Okitsu, Y. Maeda, T. A. Yamamoto, R. Oshima and Y. Nagata, J. Phys. Chem. B, 1997, 101, 7033-7037.
33 T. Iida, D. Zanchet, K. Ohara, T. Wakihara and Y. Román-Leshkov, Angew. Chem., Int. Ed., 2018, 57, 64546458.

34 X.-F. Yang, A. Wang, B. Qiao, J. Li, J. Liu and T. Zhang, Acc. Chem. Res., 2013, 46, 1740-1748.

35 M. Flytzani-Stephanopoulos and B. C. Gates, Annu. Rev. Chem. Biomol. Eng., 2012, 3, 545-574.

36 A. Datye and Y. Wang, Natl. Sci. Rev., 2018, 5, 630-632.

37 J. Jones, H. Xiong, A. T. DeLaRiva, E. J. Peterson, H. Pham, S. R. Challa, G. Qi, S. Oh, M. H. Wiebenga, X. I. Pereira Hernandez, Y. Wang and A. K. Datye, Science, 2016, 353, 150-154.

38 Z. Tang, P. Liu, H. Cao, S. Bals, H. J. Heeres and P. P. Pescarmona, ACS Catal., 2019, 9, 9953-9963.

39 S. Utamapanya, K. J. Klabunde and J. R. Schlup, Chem. Mater., 1991, 3, 175-181.

40 L. Simonelli, C. Marini, W. Olszewski, M. Ávila Pérez, N. Ramanan, G. Guilera, V. Cuartero and K. Klementiev, Cogent Phys., 2016, 3, 1231987.

41 G. Guilera, F. Rey, J. Hernández-Fenollosa and J. J. CortésVergaz, J. Phys.: Conf. Ser., 2013, 430, 12057.

42 B. Ravel and M. Newville, J. Synchrotron Radiat., 2005, 12, 537-541.

43 W. H. Cassinelli, L. Martins, A. R. Passos, S. H. Pulcinelli, C. V Santilli, A. Rochet and V. Briois, Catal. Today, 2014, 229, 114-122.

44 A. Voronov, A. Urakawa, W. van Beek, N. E. Tsakoumis, H. Emerich and M. Rønning, Anal. Chim. Acta, 2014, 840, 20-27.

45 J. Jaumot, R. Gargallo, A. de Juan and R. Tauler, Chemom. Intell. Lab. Syst., 2005, 76, 101-110.

46 J. Wei and E. Iglesia, J. Phys. Chem. B, 2004, 108, 4094-4103. 47 L. K. Ono, B. Yuan, H. Heinrich and B. R. Cuenya, J. Phys. Chem. C, 2010, 114, 22119-22133.

48 B. B. Sarma, J. Kim, J. Amsler, G. Agostini, C. Weidenthaler, N. Pfänder, R. Arenal, P. Concepción, P. Plessow, F. Studt and G. Prieto, Angew. Chem., 2020, 132, 5855-5864.

49 M. M. J. Treacy, Microsc. Microanal., 2011, 17, 847-858.

50 P. Hartel, H. Rose and C. Dinges, Ultramicroscopy, 1996, 63, 93-114.

51 Z. W. Wang, Z. Y. Li, S. J. Park, A. Abdela, D. Tang and R. E. Palmer, Phys. Rev. B Condens. Matter, 2011, 84, 73408.

52 L. O. Paz-Borbón, R. L. Johnston, G. Barcaro and A. Fortunelli, J. Phys. Chem. C, 2007, 111, 2936-2941.

53 L. O. Paz-Borbón, R. L. Johnston, G. Barcaro and A. Fortunelli, J. Chem. Phys., 2008, 128, 134517.

54 B. B. Sarma, P. N. Plessow, G. Agostini, P. Concepción, N. Pfänder, L. Kang, F. R. Wang, F. Studt and G. Prieto, J. Am. Chem. Soc., 2020, 142, 14890-14902.

55 A. Del Vitto, L. Giordano, G. Pacchioni and U. Heiz, J. Phys. Chem. B, 2005, 109, 3416-3422.

56 H. Xu, C.-Q. Xu, D. Cheng and J. Li, Catal. Sci. Technol., 2017, 7, 5860-5871.

57 Y. Zhu, K. Chen, C. Yi, S. Mitra and R. Barat, Chem. Eng. Commun., 2018, 205, 888-896.

58 J. Zhang, H. Wang and A. Dalai, J. Catal., 2007, 249, 300-310. 
59 C. Dai, S. Zhang, A. Zhang, C. Song, C. Shi and X. Guo, J. Mater. Chem. A, 2015, 3, 16461-16468.

60 R. K. Singha, A. Shukla, A. Sandupatla, G. Deo and R. Bal, J. Mater. Chem. A, 2017, 5, 15688-15699.

61 Y. Song, E. Ozdemir, S. Ramesh, A. Adishev, S. Subramanian, A. Harale, M. Albuali, B. A. Fadhel, A. Jamal, D. Moon, S. H. Choi and C. T. Yavuz, Science, 2020, 367, 777.
62 J. Wei and E. Iglesia, Angew. Chem., Int. Ed., 2004, 43, 36853688.

63 K. Klier, J. S. Hess and R. G. Herman, J. Chem. Phys., 1997, 107, 4033-4043.

64 Z. Zuo, S. Liu, Z. Wang, C. Liu, W. Huang, J. Huang and P. Liu, ACS Catal., 2018, 8, 9821-9835.

65 Y. H. Hu and E. Ruckenstein, Catal. Lett., 1997, 43, 71-77. 